\title{
Harsh default penalties lead to Ponzi schemes: A counterexample
}

\author{
V. Filipe Martins-da-Rocha ${ }^{\mathrm{a}}$, Yiannis Vailakis ${ }^{\mathrm{b}}$ \\ ${ }^{a}$ Graduate School of Economics, Getulio Vargas Foundation \\ ${ }^{b}$ University of Exeter Business School, Department of Economics
}

\begin{abstract}
Páscoa and Seghir (2009) presented two examples to show that in the presence of utility penalties for default, collateral requirements do not always eliminate the occurrence of Ponzi schemes and equilibria may fail to exist. This paper aims at providing a counterexample to their claim. We show that in the examples they consider, a competitive equilibrium with no trade can be supported due to unduly pessimistic expectations on asset deliveries.
\end{abstract}

JEL Classification: D52, D91

Keywords: Infinite horizon economies, Default penalties, Collateral, Ponzi schemes, Pessimistic expectations, No-trade

\section{Introduction}

In infinite-horizon competitive economies with full commitment, it is well-known that Ponzi schemes must be ruled out in order to guarantee the existence of equilibria. In an environment without commitment, Araujo, Páscoa and Torres-Martínez (2002) showed that Ponzi schemes are ruled out (and therefore equilibria always exist) if agents are forced to hold collateral when they take debt positions. Páscoa and Seghir (2009) subsequently presented two examples to show that if, in addition to collateral repossession, agents suffer harsh utility penalties when they default, then Ponzi schemes may reappear and equilibria fail to exist. This paper aims at showing that the economies considered in those examples do have an equilibrium with no trade.

The no trade equilibrium outcome is attributed to a simultaneous wedge at autarky between the asset price and the values of the short and long positions. More precisely, we show that it is possible to choose the asset price to lie between the value of the long position evaluated using the most pessimistic expectations about deliveries and the value of the short position in the presence of harsh default penalties. The loss of utility when defaulting is so severe that borrowers would fully repay their debts if the asset were traded. This seems to be inconsistent with lenders' pessimistic expectations about deliveries. However, the definition of an (unrefined) equilibrium does not impose any consistency condition on out of equilibrium paths. In particular, under no trade, expectations become indeterminate and the inconsistency is formally absent. 


\section{The environment}

Since our objective is to provide a counterexample to the non-existence results presented in Páscoa and Seghir (2009), we consider a specific and simple infinite horizon economy $\mathcal{E}$ without uncertainty, with one short-lived asset, one good per period and time independent primitives. The set $\{0,1, \ldots, t, \ldots\}$ of time periods is denoted by $\mathcal{T}$.

There exists one non-perishable good available for trade at every period. The depreciation factor is denoted by $Y \in(0,1)$. We interpret $x_{t} \geqslant 0$ as a claim to consumption at period $t$ and $Y x_{t-1}$ represents what is obtained at period $t$ if $x_{t-1}$ units of good are purchased at period $t-1$.

There is a finite set $I$ of infinitely lived agents. Each agent $i \in I$ is characterized by an endowment sequence $\omega^{i}=\left(\omega_{t}^{i}\right)_{t \geqslant 0}$ where $\omega_{t}^{i}>0$ denotes the endowment of the good available at period $t$. Each agent chooses a consumption sequence $x=\left(x_{t}\right)_{t \geqslant 0}$ where $x_{t} \geqslant 0$. We denote by $X$ the set of consumption sequences. The utility function $U^{i}$ is assumed to be time-additively separable, i.e., $U^{i}(x)=\sum_{t \geqslant 0}\left[\beta_{i}\right]^{t} x_{t}$ where $\beta_{i} \in(0,1)$ is the discount factor.

There is a single asset which is a short-lived real security available for trade at each period $t$, paying the dividend $A>0$ in units of the good. We let $q=\left(q_{t}\right)_{t \geqslant 0}$ be the asset price sequence where $q_{t} \geqslant 0$ represents the asset price at period $t$. For each agent $i$, we denote by $\theta_{t}^{i} \in \mathbb{R}_{+}$the purchases and by $\varphi_{t}^{i} \in \mathbb{R}_{+}$the short-sales of the asset at each period $t$. The asset is collateralized in the sense that for every unit of asset sold at a period $t$, agents should buy $C>0$ units of the good as a collateral that protects lenders in case of default. We make the assumption that the promise $A$ is strictly larger than the depreciated collateral $Y C$, i.e., there exists $b>0$ such that $A=b+Y C$. At a period $t \geqslant 1$, agent $i$ should deliver the promise $A \varphi_{t-1}^{i}$. However, agent $i$ may decide to default and choose a delivery $\Delta_{t}^{i}$ in units of the good. Since the collateral can be seized, this delivery must satisfy $\Delta_{t}^{i} \geqslant Y C \varphi_{t-1}^{i}$ (recall that $A>Y C$ ). We denote by $\sigma_{t}^{i}$ agent $i$ 's rate of repayment above the minimal delivery, i.e.,

$$
\Delta_{t}^{i}=\left[\sigma_{t}^{i} A+\left(1-\sigma_{t}^{i}\right) Y C\right] \varphi_{t-1}^{i} \quad \text { if } \quad \varphi_{t-1}^{i}>0
$$

When $\varphi_{t-1}^{i}=0$ we arbitrarily pose $\sigma_{t}^{i}=0$.

Following Dubey, Geanakoplos and Shubik (2005), Páscoa and Seghir (2009) assume that each agent $i$ feels at period $t$ a disutility from defaulting which is represented by a linear function of the extent of default. More precisely, if agent $i$ decides to deliver $\Delta_{t}^{i}$ at period $t$ given promises $\varphi_{t-1}^{i}$ made at $t-1$, then he suffers at the initial date the disutility

$$
\lambda_{t}^{i}\left[A \varphi_{t-1}^{i}-\Delta_{t}^{i}\right]^{+} \text {with } \lambda_{t}^{i}=\left[\beta_{i}\right]^{t} \mu^{i} .
$$

The parameter $\mu^{i} \in[0, \infty]$ represents the instantaneous disutility from defaulting one unit of the good. In that case, agent $i$ may have an incentive to deliver more than the minimum between his debt and the depreciated collateral, i.e., we may have $\Delta_{t}^{i}>Y C \varphi_{t-1}^{i}$ or equivalently, $\sigma_{t}^{i}>0$. The asset is thought as a pool, i.e., at each period $t$ there is a delivery rate $K_{t} \in[0,1]$ that summarizes all different sellers' deliveries. By purchasing one unit of the asset, the lenders correctly anticipate to receive the fraction $K_{t} A$. Recall that each agent $i$ delivers $\Delta_{t}^{i}=\left[\sigma_{t}^{i} A+\left(1-\sigma_{t}^{i}\right) Y C\right] \varphi_{t-1}^{i}$ with $\sigma_{t}^{i} \geqslant 0$. In particular, all 
agents rationally anticipate that $K_{t} A \geqslant Y C$ implying that there exists an aggregate rate of repayment $\sigma_{t} \in[0,1]$ satisfying

$$
K_{t} A=V_{t}(\sigma) \quad \text { where } \quad V_{t}(\sigma) \equiv \sigma_{t} A+\left(1-\sigma_{t}\right) Y C .
$$

One of the equilibrium conditions will require that lenders' expected gross return $V_{t}(\sigma)$ coincides with the actual deliveries of the borrowers in the sense that

$$
\sum_{i \in I} V_{t}(\sigma) \theta_{t-1}^{i}=\sum_{i \in I} \Delta_{t}^{i}
$$

We let $\mathcal{A}$ be the space of sequences $a=\left(a_{t}\right)_{t \geqslant 0}$ with $a_{t}=\left(x_{t}, \theta_{t}, \varphi_{t}, \Delta_{t}\right) \in \mathbb{R}_{+} \times$ $\mathbb{R}_{+} \times \mathbb{R}_{+} \times \mathbb{R}_{+} \cdot{ }^{1}$ Given a sequence $(q, \sigma)$ of asset prices and aggregate repayment rates, we denote by $B^{i}(q, \sigma)$ the set of agent $i$ 's choices $a^{i}=\left(x^{i}, \theta^{i}, \varphi^{i}, \Delta^{i}\right) \in \mathcal{A}$ satisfying the following constraints:

(a) solvency: $x_{t}^{i}+\Delta_{t}^{i}+q_{t} \theta_{t}^{i} \leqslant\left[\omega_{t}^{i}+Y x_{t-1}^{i}\right]+V_{t}(\sigma) \theta_{t-1}^{i}+q_{t} \varphi_{t}^{i}$;

(b) collateral requirement: $C \varphi_{t}^{i} \leqslant x_{t}^{i}$ and minimum delivery: $Y C \varphi_{t-1}^{i} \leqslant \Delta_{t}^{i}$.

When choosing $a=(x, \theta, \varphi, \Delta) \in \mathcal{A}$, agent $i$ gets the utility $U^{i}(x)=\sum_{t \geqslant 0}\left[\beta_{i}\right]^{t} x_{t}$ but suffers the disutility $W^{i}(a)=\sum_{t \geqslant 1}\left[\beta_{i}\right]^{t} \mu^{i}\left[A \varphi_{t-1}-\Delta_{t}\right]^{+}$. We would like to define agent $i$ 's payoff $\Pi^{i}(a)$ as $U^{i}(x)-W^{i}(a)$. Unfortunately, this difference may not be well-defined if both $U^{i}(x)$ and $W^{i}(a)$ are infinite. We propose to consider the binary relation $\succ_{i}$ defined on $\mathcal{A}$ by

$$
\widetilde{a} \succ_{i} a \Longleftrightarrow \exists \varepsilon>0, \quad \exists T \in \mathcal{T}, \quad \forall t \geqslant T, \quad \Pi^{i, t}(\widetilde{a}) \geqslant \Pi^{i, t}(a)+\varepsilon
$$

where $\Pi^{i, t}(a)=U^{i, t}(x)-W^{i, t}(a)$ with $U^{i, t}(x)=\sum_{0 \leqslant \tau \leqslant t}\left[\beta_{i}\right]^{\tau} x_{\tau}$ be the truncated utility, and $W^{i, t}(a)=\sum_{1 \leqslant \tau \leqslant t}\left[\beta_{i}\right]^{\tau} \mu^{i}\left[A \varphi_{\tau-1}-\Delta_{\tau}\right]^{+}$be the truncated disutility. Observe that if $\Pi^{i}(\widetilde{a})$ and $\Pi^{i}(a)$ exist in $\mathbb{R}$ then $\widetilde{a} \succ_{i} a$ if and only if $\Pi^{i}(\widetilde{a})>\Pi^{i}(a)$. The set $\operatorname{Pref}^{i}(a)$ of plans strictly preferred to plan $a$ by agent $i$ is defined by $\operatorname{Pref}^{i}(a)=\left\{\widetilde{a} \in \mathcal{A}: \widetilde{a} \succ_{i} a\right\}$.

We denote by $\Xi$ the set of sequences of prices and aggregate repayment rates $(q, \sigma)$ such that $\left(q_{t}, \sigma_{t}\right) \in \mathbb{R}_{+} \times[0,1]$ for all $t \geqslant 0$. A competitive equilibrium is a family of prices and aggregate repayment rates $(q, \sigma) \in \Xi$ and an allocation $\boldsymbol{a}=\left(a^{i}\right)_{i \in I}$ with $a^{i} \in \mathcal{A}$ such that: for every agent $i$, the plan $a^{i}$ is optimal among the budget feasible plans, i.e.,

$$
a^{i} \in B^{i}(q, \sigma) \text { and } \operatorname{Pref}^{i}\left(a^{i}\right) \cap B^{i}(q, \sigma)=\emptyset ;
$$

the good market clears at every period, i.e., ${ }^{2}$

$$
\forall t \geqslant 0, \quad \sum_{i \in I} x_{t}^{i}=\sum_{i \in I}\left[\omega_{t}^{i}+Y x_{t-1}^{i}\right]
$$

\footnotetext{
${ }^{1}$ By convention we let $a_{-1}=\left(x_{-1}, \theta_{-1}, \varphi_{-1}, \Delta_{-1}\right)=(0,0,0,0)$.

${ }^{2}$ By convention $x_{-1}^{i}=0$ and $Y_{-1}=0$.
} 
the asset market clears at every period, i.e.,

$$
\forall t \geqslant 0, \quad \sum_{i \in I} \theta_{t}^{i}=\sum_{i \in I} \varphi_{t}^{i}
$$

aggregate borrowers' deliveries match lenders' expectations, i.e.,

$$
\forall t \geqslant 1, \quad \sum_{i \in I} V_{t}(\sigma) \theta_{t-1}^{i}=\sum_{i \in I} \Delta_{t}^{i} .
$$

For each agent $i$, we denote by $\Omega^{i}=\left(\Omega_{t}^{i}\right)_{t \geqslant 0}$ the sequence of accumulated endowments, defined recursively by $\Omega_{t}^{i}=Y_{t} \Omega_{t-1}^{i}+\omega_{t}^{i}$ with $\Omega_{0}^{i}=\omega_{0}^{i}$. We assume that accumulated endowments are uniformly bounded from above, i.e., there exists $\Omega_{\text {sup }}^{i}>0$ such that $\Omega_{t}^{i} \leqslant \Omega_{\text {sup }}^{i}$ for every $t \in \mathcal{T}$. The sequence $\sum_{i \in I} \Omega^{i}$ of accumulated aggregate endowments is denoted by $\Omega$.

\section{Indeterminacy of repayment rates and pessimistic expectations}

Let $\left(\pi,\left(a^{i}\right)_{i \in I}\right)$ be a competitive equilibrium with prices $\pi=(q, \sigma) \in \Xi$ and plans $a^{i}=\left(x^{i}, \theta^{i}, \varphi^{i}, \Delta^{i}\right)$. Fix a period $t \geqslant 1$. If there is trade in period $t-1$, i.e., $\varphi_{t-1}^{i}>0$ for some agent $i$, then equation (4) in the definition of a competitive equilibrium can be restated as follows

$$
\sigma_{t} \sum_{i \in I} \varphi_{t-1}^{i}=\sum_{i \in I} \sigma_{t}^{i} \varphi_{t-1}^{i}
$$

and $\sigma_{t}$ can be interpreted as the average repayment rate (per unit of asset sold) above the minimum delivery $Y C$. If there is no trade in period $t-1$ then the repayment rate $\sigma_{t}$ is undeterminate. That is, when the asset is not traded, our equilibrium concept makes no assumption about the expected repayment rate.

We claim that pessimistic expectations about repayments (i.e., low values of $\sigma_{t}$ ) may by itself render the asset market inactive in period $t-1$ if default penalties are large enough. Our finding shares some similarities with the issue of trivial equilibria pointed out by Dubey et al. (2005). To clarify this link, recall that the delivery rate, denoted by $K_{t}$, is defined by the equation

$$
K_{t} A \sum_{i \in I} \varphi_{t-1}^{i}=\sum_{i \in I} \Delta_{t}^{i}
$$

As explained in Páscoa and Seghir (2009) (see Remark 3.1), when assets are collateralized agents deliver at least $Y C$ per unit of asset sold. In this case, rational agents expect $K_{t}$ to be greater than the ratio $Y C / A$, and in particular it must be non-null. This is the reason why in our presentation of the model we have chosen to parameterize agents' expectations about delivery by the average repayment rate above the minimum delivery, denoted by $\sigma_{t}$. In other words, when there is trade in period $t-1$ we have the relation

$$
K_{t} A=\sigma_{t} b+Y C .
$$

In Dubey et al. (2005) there are no durable goods (say $Y=0$ ) and assets are not collateralized. In such a framework, it is easy to support equilibria with no trade in the 
asset on account of absurdly pessimistic expectations about delivery rates (i.e., $K_{t}=0$ ). However, in a model with collateral requirements, it is not clear whether such equilibria can be supported since we always have $K_{t} \geqslant Y C / A>0 .^{3}$ Páscoa and Seghir (2009) claimed that trivial no-trade equilibria do not exist when the securities are collateralized. The contribution of this paper is to show that although agents expect delivery rates $K_{t}$ to be strictly positive (actually above or equal to $Y C / A$ ) there is still room for unduly pessimistic expectations that sustain equilibrium with no trade. To illustrate our point we consider the two examples presented by Páscoa and Seghir (2009) and show that when default penalties are harsh enough no trade is a possible equilibrium outcome. A direct implication of our finding is that in those examples harsh default penalties do not always lead to Ponzi schemes as it is claimed by Páscoa and Seghir (2009).

Choose the family $\left(\beta_{i}\right)_{i \in I}$ such that the following inequality holds true

$$
\beta_{\max } Y C<\min \left\{C, \beta_{\min } A\right\}
$$

where $\beta_{\min }=\min _{i \in I} \beta_{i}$ and $\beta_{\max }=\max _{i \in I} \beta_{i}{ }^{4}$

Theorem. For every depreciation factor $Y$ small enough and every family $\left(\mu^{i}\right)_{i \in I}$ of instantaneous default penalties satisfying

$$
\forall i \in I, \quad \mu^{i} \geqslant \frac{1}{1-\beta_{i} Y}
$$

the no trade allocation $\left(\text { aut }^{i}\right)_{i \in I}$ with aut ${ }^{i}=\left(\Omega^{i}, 0,0,0\right)$ is a competitive equilibrium under the price and aggregate repayment rates system $\pi=\left(q_{t}, \sigma_{t}\right)_{t \in \mathcal{T}}$ defined by $\sigma_{t}=0$ and $q_{t}=q$ where $q$ can be suitably chosen to satisfy

$$
\beta_{\max } Y C<q<\min \left\{C, \beta_{\min } A\right\} .
$$

Remark 1. Observe that $\sigma_{t}=0$ corresponds to $K_{t}=Y C / A>0$. That is, under this price system, no trade is a non-trivial equilibrium according to Definition 3.3 in Páscoa and Seghir (2009).

Our theorem illustrates that even if default penalties are harsh and lenders' expect asset deliveries to be strictly positive, pessimistic expectations destroy incentives to trade

\footnotetext{
${ }^{3}$ The intuition behind the existence of trivial equilibria in Dubey et al. (2005) is as follows. Consider the no-trade (autarky) allocation. Introduce next an asset in period $t$. Choose the delivery rate $K_{t+1}$ of the asset equal to zero and the price $q_{t}$ equal to zero. Then no agent would have an incentive to trade. In a model with collateralized obligations this argument breaks down since $K_{t+1}$ must be larger that $Y C / A$. One may try to implement no trade by choosing $\sigma_{t+1}=0$ (or equivalently $K_{t+1}=Y C / A$ ) and fixing the asset price $q_{t}=C$. No agent would then have incentives to invest. Indeed, it would be better to buy $C$ units of the good instead of one unit of the asset because of the utility obtained from consuming the collateral. However, it is not clear whether agents would have no incentives to sell the asset. It depends on whether the gain from consuming the collateral in period $t$ can compensate the future penalty suffered in case of default or the loss in consumption due to the repayment of debt besides the value of the depreciated collateral.

${ }^{4}$ It is always possible to find a family $\left(\beta_{i}\right)_{i \in I}$ satisfying (5) since $A>Y C$ and $Y<1$. In particular, one may consider an economy with a single agent. In that case, the above inequality is satisfied for any discount factor $\beta \in(0,1)$.
} 
and no trade can be supported as a competitive equilibrium. Before providing the rigorous arguments to prove the above theorem, we provide some intuition to understand why the no trade plan $\left(\mathrm{aut}^{i}\right)$ is optimal among budget feasible plans.

Since $q>\beta_{i} Y C$ the asset is too expensive to provide incentives to invest. Indeed, lenders have pessimistic expectations and believe that the unitary payment of the asset will be $Y C$ (which corresponds to the minimum delivery associated to the seizure of collateral). Fix a period $t$ and consider that agent $i$ decides to buy $\varepsilon>0$ units of the asset (with $\varepsilon>0$ small). He has to reduce his current consumption by $q \varepsilon$. Since the good is durable, the agent will also have to reduce his consumption at $t+1$ by $Y q \varepsilon$. In a similar way, his consumption at $t+2$ will have to be reduced by $Y^{2} q \varepsilon$ and so on. The overall impact in terms of utility (at period $t$ ) is a decrease of $q \varepsilon\left(1+\beta_{i} Y+\left(\beta_{i} Y\right)^{2}+\ldots\right)=$ $q \varepsilon /\left(1-\beta_{i} Y\right)$. The purchase of $\varepsilon$ units of the asset at period $t$ implies a delivery $\beta_{i} Y C \varepsilon$ at $t+1$. The overall impact in terms of utility (at period $t$ ) of this delivery is an increase of $\beta_{i} Y C \varepsilon /\left(1-\beta_{i} Y\right)$ which does not compensate the loss $q \varepsilon /\left(1-\beta_{i} Y\right)$.

Since $q<\beta_{i} A$, the asset price is too low to provide incentives to take a short position. Indeed, default is prevented since the penalty coefficient $\mu^{i}$ is higher than the marginal "overall" utility of consumption $1 /\left(1-\beta_{i} Y\right) .{ }^{5}$ If agent $i$ sells $\varepsilon>0$ units of the asset at period $t$, he will have to repay the full amount $A \varepsilon$ at $t+1$. The increase of overall utility $q \varepsilon /\left(1-\beta_{i} Y\right)$ due to the increase of consumption does not compensate the decrease of overall utility $\beta_{i} A \varepsilon /\left(1-\beta_{i} Y\right)$ due to repayment at $t+1$ (and consequently the decrease of consumption at subsequent periods since the good is durable).

These two conditions $\left(\beta_{i} Y C<q\right.$ and $\left.q<\beta_{i} A\right)$ ensure that the no trade plan $\left(\mathrm{aut}^{i}\right)$ is optimal among finite horizon allocations. The fact that $q<C$ will enable us to prove that any budget feasible plan satisfies a transversality condition, which ensures the optimality of the no trade plan among all budget feasible plans.

Remark 2. The economy described above satisfies all the conditions of the two (classes of) examples of non-existence proposed by Páscoa and Seghir (2009). In their first example, the unitary default penalty $\mu^{i}$ is chosen to be large enough, in particular it can be taken to satisfy (6). In the second example, $\mu^{i}$ is assumed to satisfy

$$
\mu^{i}=\frac{f^{i}(\# I)}{(1-Y)\left(1-\beta_{i}\right)}
$$

for some $f^{i}>1$. Observe that such default penalties satisfy condition (6) in our theorem.

\section{Proof of the theorem}

The proof of the theorem is split in three steps: (1) we show optimality of aut ${ }^{i}$ among budget feasible plans with a finite horizon; (2) we prove that aut $^{i}$ is optimal among budget feasible plans with finite utility; and finally (3) we show that any plan $a=(x, \theta, \varphi, \Delta)$ in the budget set $B^{i}(\pi)$ has a finite utility.

Step 1. We first show that first order conditions are satisfied for some well-chosen Lagrange multipliers. For any agent $i$ we should find a sequence $\left(\delta_{t}^{i}\right)_{t \in \mathcal{T}}$ of super-gradients

\footnotetext{
${ }^{5}$ Recall that the good is durable.
} 
of $z \mapsto[z]^{+}$at 0 , i.e., any real number in $[0,1]$ and a sequence $\left(\gamma_{t}^{i}, \rho_{t}^{i}, \chi_{t}^{i}, \alpha_{\theta, t}^{i}, \alpha_{\varphi, t}^{i}\right)_{t \in \mathcal{T}}$ of non-negative Lagrange multipliers ${ }^{6}$ such that first order conditions (henceforth foc) are satisfied:

(a) foc for consumption: $\left[\beta_{i}\right]^{t}+\gamma_{t+1}^{i} Y+\chi_{t}^{i}=\gamma_{t}^{i}$ for every $t \geqslant 0$;

(b) foc for asset purchases: $\gamma_{t+1}^{i} Y C+\alpha_{\theta, t}^{i}=\gamma_{t}^{i} q$ for every $t \geqslant 0$;

(c) foc for deliveries: $\left[\beta_{i}\right]^{t} \mu^{i} \delta_{t}^{i}+\rho_{t}^{i}=\gamma_{t}^{i}$ for every $t \geqslant 1$;

(d) foc for asset sales: $\gamma_{t}^{i} q+\alpha_{\varphi, t}^{i}=\rho_{t+1}^{i} Y C+\chi_{t}^{i} C+\beta_{i}^{t+1} \mu^{i} \delta_{t+1}^{i} A$ for every $t \geqslant 1$;

(e) binding restrictions at the plan $\left(x^{i}, \theta^{i}, \varphi^{i}, \Delta^{i}\right)=\left(\operatorname{aut}^{i}\right): \chi_{t}^{i}\left[x_{t}^{i}-C \varphi_{t}^{i}\right]=0, \rho_{t}^{i}\left[\Delta_{t}^{i}-\right.$ $\left.Y C \varphi_{t-1}^{i}\right]=0, \alpha_{\theta, t}^{i} \theta_{t}^{i}=0, \alpha_{\varphi, t}^{i} \varphi_{t}^{i}=0$ and

$$
\gamma_{t}^{i}\left[\Omega_{t}^{i}+Y C \theta_{t-1}^{i}+q\left(\varphi_{t}^{i}-\theta_{t}^{i}\right)-\Delta_{t}^{i}-x_{t}^{i}\right]=0 .
$$

We propose to make the following choices. Since $\varphi_{t}^{i}=0$ we have $x_{t}^{i}=\Omega_{t}^{i}>C \varphi_{t}^{i}$ and therefore the Lagrange multiplier $\chi_{t}^{i}$ associated to the collateral requirement must satisfy $\chi_{t}^{i}=0$. We pose $\rho_{t}^{i}=0, \delta_{t}^{i}=\delta^{i}$ for some $\delta^{i} \in[0,1]$ and $\gamma_{t}^{i}=\left[\beta_{i}\right]^{t} \mu^{i} \delta^{i}$. We then get the first order conditions for deliveries. We choose $\delta^{i}$ such that $1=\mu^{i} \delta^{i}\left[1-\beta_{i} Y\right]$. Since $\mu^{i}$ is assumed to be larger than $1 /\left(1-\beta_{i} Y\right)$, we get that $\delta^{i}$ belongs to $[0,1]$. It follows that first order conditions for consumption are satisfied. We let $\alpha_{\theta, t}^{i}=\left[\beta_{i}\right]^{t} \mu^{i} \delta^{i}\left[q-\beta_{i} Y C\right]$ and get the first order condition for asset purchases. We let $\alpha_{\varphi, t}^{i}=\left[\beta_{i}\right]^{t} \mu^{i} \delta^{i}\left[\beta_{i} A-q\right]$ and then get the first order condition for asset sales. Since $\theta_{t}^{i}=0$ for every $t$, first order conditions ensure optimality among budget feasible plans with a finite horizon.

In the remaining two steps we prove that the no trade plan aut ${ }^{i}$ is indeed optimal among all budget feasible plans. In step (2) it is shown that aut ${ }^{i}$ is optimal among budget feasible plans with finite utility, while in step (3) we show that any plan $a=(x, \theta, \varphi, \Delta)$ in the budget set $B^{i}(\pi)$ has a finite utility.

Step 2. Consider a budget feasible plan $a=(x, \theta, \varphi, \Delta)$ with $U^{i}(x)$ finite and such that $\Pi^{i}(a)>\Pi^{i}\left(\right.$ aut $\left.^{i}\right)$. Given a period $\tau>0$, we let $a^{\tau}$ be the "truncated" plan defined by $a_{t}^{\tau}=a_{t}$ if $t \leqslant \tau, a_{t}^{\tau}=(0,0,0,0)$ if $t>\tau+1$ and $a_{t}^{\tau}=\left(x_{t}-C \varphi_{t}, 0,0, \Delta_{t}\right)$ if $t=\tau+1$. Since $C>q$ the plan $a^{\tau}$ is budget feasible. Moreover, for $\tau$ large enough we have $\Pi^{i}\left(a^{\tau}\right)>\Pi^{i}\left(\right.$ aut $\left.^{i}\right)$ which contradicts optimality among budget feasible plans with a finite horizon.

Step 3. We propose to conclude the proof by showing that any plan $a=(x, \theta, \varphi, \Delta)$ in the budget set $B^{i}(\pi)$ has a finite utility. Actually, we will prove that the sequence $\left(x_{t}\right)_{t \in \mathcal{T}}$ is uniformly bounded from above. To see this, we let $\widehat{x}_{t}=x_{t}-C_{t} \varphi_{t} \geqslant 0$. From the budget constraint at $t=0$, we have

$$
\widehat{x}_{0}+q \theta_{0}+(C-q) \varphi_{0} \leqslant \omega_{0}^{i} \leqslant \Omega_{\text {sup }}^{i}
$$

\footnotetext{
${ }^{6}$ The Lagrange multiplier $\gamma_{t}^{i}$ corresponds to the budget constraint, $\rho_{t}^{i}$ to the minimum delivery constraint, $\chi_{t}^{i}$ to the collateral requirement, $\alpha_{\theta, t}^{i}$ and $\alpha_{\varphi, t}^{i}$ to the non-negative constraints on portfolio purchases and sales.
} 
At $t=1$, we have

$$
\widehat{x}_{1}+q \theta_{1}+(C-q) \varphi_{1} \leqslant \omega_{1}^{i}+Y x_{0}+Y C \theta_{0} \leqslant \Omega_{\text {sup }}^{i}+Y x_{0}+Y C \theta_{0} .
$$

Observe that

$$
\begin{aligned}
x_{0}+C \theta_{0} & =\widehat{x}_{0}+q \theta_{0}+(C-q) \varphi_{0}+(C-q) \theta_{0}+q \varphi_{0} \\
& \leqslant \Omega_{\mathrm{sup}}^{i}+(C-q) \frac{\Omega_{\mathrm{sup}}^{i}}{q}+q \frac{\Omega_{\mathrm{sup}}^{i}}{C-q} \\
& \leqslant \Omega_{\mathrm{sup}}^{i}\left[\frac{C}{q}+\frac{q}{C-q}\right] .
\end{aligned}
$$

We have thus proved that $\widehat{x}_{1}+q \theta_{1}+(C-q) \varphi_{1} \leqslant \Omega_{\text {sup }}^{i}[1+\aleph]$ where

$$
\aleph \equiv \frac{Y C}{q}+\frac{Y q}{C-q}=\frac{Y C}{q}+\frac{Y}{C / q-1} .
$$

Actually we can prove recursively that

$$
\widehat{x}_{t}+q \theta_{t}+(C-q) \varphi_{t} \leqslant \Omega_{\sup }^{i}\left[1+\aleph+\aleph^{2}+\ldots+\aleph^{t}\right] .
$$

Recall that we propose to choose $q$ satisfying (7), i.e., $\beta_{\max } Y C<q<\min \left\{C, \beta_{\min } A\right\}$. Fix $\Gamma>1 / \beta_{\max }$ and choose $q=\widehat{q}(Y)=\Gamma \beta_{\max } Y C$. Since $\beta_{\max }<1$ we have $\Gamma>1$ and we automatically get $\beta_{\max } Y C<\widehat{q}(Y)$. Choosing $Y$ small enough we get the other inequality $\widehat{q}(Y)<\min \left\{C, \beta_{\min } A\right\}$. Given the choice of $q$ we have

$$
\aleph=\frac{1}{\Gamma \beta_{\max }}+\Gamma \beta_{\max } \frac{Y^{2}}{1-\Gamma \beta_{\max } Y} .
$$

Choosing $Y$ small enough, we get that $\aleph \in(0,1)$. Therefore if $a=(x, \theta, \varphi, \Delta)$ belongs to the budget set $B^{i}(\pi)$ then the consumption process is uniformly bounded from above. More precisely we have $x_{t} \leqslant \Omega_{\text {sup }}^{i} /(1-\aleph)$.

Remark 3. Observe that the above argument is based on a very peculiar property of our example: there is a uniform lower bound on the "hair cut" $C-q_{t}$. This is fine since our aim is to present a counterexample, but it should be stressed that this is far from being a general feature of models with collateral.

\section{Concluding remarks}

For the two examples proposed in Páscoa and Seghir (2009), we have shown that there exists a no trade equilibrium due to pessimistic expectations about repayment rates. Our result raises an interesting issue: the equilibrium concept should be refined in order to rule out such pathological no trade equilibria.

In a companion working paper (see Martins-da-Rocha and Vailakis (2011)) we show than we can adapt the refinement procedure introduced by Dubey et al. (2005) to eliminate "undesirable" no-trade equilibria. It is then possible to prove that harsh default penalties preclude existence of "refined" equilibria. In other words, our counter-example does not change the overall message of Páscoa and Seghir (2009), but does show that their arguments and assumptions are not quite sufficient to prove their claims. 


\section{References}

Araujo, A.P., Páscoa, M.R., Torres-Martínez, J.P., 2002. Collateral avoids Ponzi schemes in incomplete markets. Econometrica 70, 1613-1638.

Dubey, P., Geanakoplos, J., Shubik, M., 2005. Default and punishment in general equilibrium. Econometrica $73,1-37$.

Martins-da-Rocha, V.F., Vailakis, Y., 2011. On Ponzi schemes in infinite horizon collateralized economies with default penalties. Economic Essays, Getulio Vargas Foundation.

Páscoa, M.R., Seghir, A., 2009. Harsh default penalties lead to Ponzi schemes. Games and Economic Behavior 65, 270-286. 